\title{
Comparative Study of Deflated Balloon Facilitation versus Balloon Dilatation before Primary PCI
}

\author{
H.M.Abo Elenin, M.A.Hamouda, H.A.Khames, A.H.Abdel Kader and M.M.Ali \\ Cardiology Dept., Faculty of Medicine, Benha Univ., Benha, Egypt \\ E-Mail:ahmed56@gmail.com
}

\begin{abstract}
Primary percutaneous coronary intercession (PPCI) remains the treatment of decision since it is related with a huge decrease in bleakness and mortality. The points of this investigation is to analyze between collapsed inflatable and swell dilatation prior to stenting during essential PCI. The current investigation was relative examination that incorporate 60 patients determined to have STEMI and went through PPCI. Gathering 1 comprise of 30 patients went through flattened inflatable encouraged direct stenting DBDS technique.Group 2 comprise of 30 patients went through inflatable swelling prior to stenting. . The MACE rate during the collapsed expand method was essentially lower than MACE rate in inflatable swelling gathering $(3.3 \%$ Vs $26.7 \% \mathrm{P}$ value=0.011). The inconvenience rate was $13.3 \%$ in the emptied expand gathering while it was $43.3 \%$ in the inflatable expansion group. The no reflow rate and distal embolization rate after DBDS strategy was $6.7 \%$ while it was $26.7 \%$ after inflatable swelling procedure. The cardiovascular breakdown rate happened distinctly in one patient in the DBDS gathering while it happened in 6 patients in the inflatable swelling gathering. The DBDS method to encourage DS has all the earmarks of being basic, possible and safe. It fundamentally builds DS and could offer an option in contrast to PD and thrombectomy.
\end{abstract}

Keywords: Deflated Balloon, Balloon Facilitation, Primary PCI.

\section{Introduction}

ST-elevation myocardial dead tissue (STEMI) is described by thrombotic impediment of the coronary vein accelerated by plaque crack. Essential percutaneous coronary intercession (PPCI) remains the treatment of decision since it is related with a huge decrease in grimness and mortality [1,2].

Most methods are presently performed with drugeluting stents, which are related with a lower pace of restenosis than uncovered metal stents. Distal embolization during PCI is a significant supporter of microvascular injury, bringing about tenacious STportion changes, bigger infarct size, hemodynamic precariousness and demise [3].

Pharmacologically encouraged PCI has brought about expanded draining danger and neglected to give upgrades in clinical results [4]. Inflatable expansion is related with higher danger of distal embolization and microvascular impediment, which is related with more terrible results [5].

In addition, ongoing randomized clinical preliminaries and meta-examinations indicated that the standard utilization of goal thrombectomy in STEMI was not related with any advantage on momentary results [6].

Thinking about this, direct stenting has all the earmarks of being the current most ideal alternative for PPCI. The accessibility of low profile stent conveyance frameworks has additionally given force to stenting. Studies have indicated that DS abbreviates procedural time, lessens radiation and difference presentation and has lower antagonistic occasion rate [7].

To perform DS, it is obligatory to picture the length of offender sore and the measurement of the downstream conduit. Lamentably, as a rule of STEMI, the thrombolysis in myocardial dead tissue (TIMI) stream remains $\leq 1$ after wire position. Thusly, the practicality of DS has been very low going from $30 \%$ to half. We used a flattened inflatable to encourage DS by representation of guilty party injury, in patients with all out impediment of the offender vein.

The points of this examination is to look at between flattened inflatable and inflatable dilatation prior to stenting during essential PCI.

\section{Patient and method}

This study is a forthcoming, controlled examination that will incorporate 60 patients introduced by ST section height myocardial dead tissue conceded at Banha clinic, October 6 college clinic, Dar El fouad Hospital in the period between January 2018 and December 2019. 30cases did an inflatable dilatation and 30 cases did flattened inflatable strategy prior to stenting during PPCI.

\section{Incorporation standards}

1. Patient age $>18$ years who grumble of common chest agony and ECG indicative of STEMI inside $12 \mathrm{~h}$ of manifestation beginning or somewhere in the range of 12 and $24 \mathrm{~h}$ on the off chance that they had constant side effects with proof of continuous ischemia, were remembered for the investigation for PPCI.

\section{Prohibition models}

1. Patients who gave heart failure, with reperfusion delay $>24 \mathrm{~h}$ and additionally not giving assent for the technique were prohibited.

2. Patients with AF, atrial ripple, or complete heartblock, cardiogenic stun, 3. Rheumatic heart infections . 4. Any patient did blood clot goal

All patients gave a finding of intense ACS STEMI along these lines subsequent to affirming the determination by ECG. A full labs were removed including first set cardiovascular proteins and troponin, 
renal capacity, liver capacity and complete blood picture while doing an ECG. Precise observing of the patients by compact screens for proceed with ECG, BP, heartbeat and oxygen immersion checking in ER and during tolerant transportation to the cath lab. Oxygen uphold through nasal canula or breathing apparatus was given if oxygen immersion was under 90 and $A B G$ test was separated quickly. Double antiplatelets treatment including stacking portion of asprin $300 \mathrm{mg}$ and ticagrelor $180 \mathrm{mg}$ or clopidogrel $300 \mathrm{mg}$ were taken. The patients sign an assent structure for essential PCI subsequent to explaining the dangers and advantages of the technique. All patients were educated about the examination system and follow up arrangement and all acknowledged it. All methods were performed inside $60 \mathrm{~min}$ from the principal clinical contact. Spiral methodology was acted in 35 patients and femoral methodology were acted in 25 patients.

Gathering 1 was incorporate 30 patients will go through 1ry PCI with the utilization of emptied expand. Patients with complete impediment of the vessel after wire position were exposed to emptied expand encouraged DS procedure (DBDS method). In this procedure, a semi-agreeable inflatable was taken with width $(2-2.5) \mathrm{mm}$ and length (15-20) $\mathrm{mm}$. It was delicately crossed past the injury in collapsed state and pulled once more into the directing catheter. From that point, a little (2 - $3 \mathrm{~mL}$ of difference specialist) infusion was taken to survey the distal TIMI grade stream. On the off chance that TIMI stream $\geq 1$ was accomplished and distal finish of injury was pictured, stenting was finished. In the event that stream couldn't be accomplished, the inflatable intersection was rehashed with contrast infusions to a limit of multiple times. As a rule, the distal stream would keep going for few moments; in this way, a prompt visual assessment of injury or fluoroscopy snatch picture was utilized for stenting. In the event that stream actually remained $\leq$ TIMI 1, a similar inflatable was progressed across the injury and utilized for pre-dilatation.

Gathering 2 was incorporate 30 patients, all patients will go through 1ry PCI with expand swelling prior to stenting. After the methodology the outspread sheath eliminated and a TR band applied to control draining and forestall hematoma development. The TR band eliminated after progressive emptying of its inflatable and check for any dying, it my discussion 2-3 hours after the method.

If there should be an occurrence of femoral methodology the sheath will be eliminated following 6 hours if no inconvenience happens and all patients were educated to stay away from any hip flexion development until 4-6hrs after expulsion of the femoral sheath. The femoral sheath and cut site consistently checked by the specialists and medical caretakers in control.

In the CCU all patients were urged to drink bounty measure of liquids. The patients were firmly checked with sufficient computation of liquid equilibrium and Vital signs. ECG performed after the strategy and at regular intervals notwithstanding 3 arrangements of cardiovascular enzymrs and toponin each 8hrs and echocardiography

\section{Essential end point incorporates}

I) TIMI stream: This TIMI arrangement was created by the TIMI (Thrombolysis in Myocardial Infarction) study gathering to semiquantitatively survey coronary vein perfusion past purpose of impediment on coronary angiography.PAMI (Primary Angioplasty in Myocardial Infarction) agents precised the portrayal of TIMI 3 as "opacification of the vessel inside three cardiovascular cycles).

II) Myocardial redden: An angiographic term alluding to the sufficiency of perfusion and blood course through a zone served by a coronary corridor, the more drawn out the become flushed persevere, the less fortunate the blood stream.

\section{Optional end focuses incorporate}

I) MACE during strategy and multi month later incorporate Reinfarction, Rehospitalization, Death from cardiovascular and non heart causes and Bleeding.

II) Ejection division by Echocardiography.

\section{Results}

This study selected sixty patients from Banha college medical clinic, DAR ELFOAD medical clinic, October 6 college medical clinic who are griping of run of the mill chest torment, determined to have STEMI and did essential PCI.

The Deflated inflatable patients incorporate 9 patients $(30.0 \%)$ are female while $21(70.0 \%)$ patients are guys. The period of patients range from 44-71 years with the mean age rises to $58.63 \pm 8.02$.

12 patients are hypertensive (40\%), 17 patients are diabetic $(56.7 \%)$ and 8 patients is experiencing dyslipidemia (26.7\%).16 patients are smoker $(53.3 \%)$ while 14 patients are non smoker $(46.7 \%)$. The inflatable swelling patients include:n13 patients (43.3 $\%$ ) are female while $17(56.7 \%$ ) patients are guys.

The period of patients range from 45-74 years with the mean age approaches $60.00 \pm 8.17 .17$ patients are hypertensive $(56.7 \%), 18$ patients are diabetics $(60.0 \%)$ and 11 patients is experiencing dyslipidemia (36.7\%).

13 patients are smoker $(43.3 \%)$ while 16 patient are non smoker $(53.3 \%)$ and 1 patient is an Ex smoker $(3.3 \%)$. In the collapsed expand bunch 4 patient have a previous history of ischemic heart infections (13.3\%)which incorporate 3 patients with past PCI of other coronary veins and one patient has a background marked by cerebrovascular stroke.

7 patients have a family background of ischemic heart illnesses $(23.3 \%)$.

The overall assessment of 24 patients were typical (80.0\%), while 2 patient were corpulent $(6.7 \%)$ and one patient had neurological shortfall as gentle muscle shortcoming in left half of the body $(3.3 \%)$. In the inflatable swelling bunch 7patient have a previous 
history of ischemic heart infections (23.3\%) which incorporate patients with past PCI of other coronary veins.

6 patients have a family background of ischemic heart sicknesses $(20.0 \%)$

The overall assessment of 27 patients were typical $(90.0 \%)$, while 5 patient were corpulent $(16.7 \%)$ and one patient has rheumatoid joint pain $(3.3 \%)$.

The mean EF during hospitalization in the emptied expand bunch was $49.47 \pm 8.36$ while in the inflatable swelling bunch was $46.83 \pm 10.11$

EF at follow up following multi month was $53.63 \pm$ 6.65 in the flattened inflatable gathering and $52.53 \pm$ 5.92 in the inflatable expansion gathering. this demonstrated non critical factual distinction Mean creatinine levels were $0.95(0.8-1.1)$ in flattened inflatable gathering and $1(0.9-1.12)$ in inflatable swelling gathering. This demonstrated non critical measurable distinction.

In Deflated inflatable patients, Hb level was typical in 28 patients $(93.3 \%$ ) and was high in 2 patient (6.7\%)while in the inflatable expansion bunch $\mathrm{Hb}$ level was ordinary in 27 patient $(90.0 \%)$ and high in 3 patients $(10.0 \%)$ this demonstrated non huge measurable distinction. Complete leucocytic includes were typical in all patients of the two gatherings.

In Deflated inflatable patients, LFT results were typical in 29patients $(96.7 \%)$ and high in 1patient $(3.3 \%)$ while in the inflatable swelling bunch LFT results were ordinary in 26 patient $(86.7 \%)$ and high in 4 patients $(13.3 \%)$ this indicated non critical factual contrast.

In the collapsed expand bunch as respect scope of pinnacle CKMB levels after affirmation equivalent 114 - 998, middle $271(210-370)$ while in the inflatable swelling bunch equivalent 66 - 1200, middle 290.5 (153 - 453) this demonstrated non huge factual distinction.

In the collapsed expand bunch as respect scope of CKMB levels at release approaches range $21-340$, middle $110.5(70-150)$ while in the inflatable swelling bunch rises to $34-700$, middle 109 (66 - 214).), this demonstrated non critical factual contrast. The pinnacle troponin levels range equivalent 889 - 12100 middle (IQR)2385 (1988 - 4490) in the emptied swell gathering while The pinnacle troponin levels in inflatable expansion bunch range equivalent 970 8180,median (IQR) 3034.5 (1799 - 5037), this indicated non huge factual distinction

The release troponin levels range equivalent 2848404 middle (IQR) $1189.5(714$ - 1880)) in the emptied swell gathering while the pinnacle troponin levels range equivalent $340-6000$, middle (IQR) 1588.5 (896 - 2478), this demonstrated non critical measurable contrast. The spans of inflatables utilized during the examination demonstrated non huge measurable contrast.

In the flattened inflatable gathering the TIMI stream after the strategy range1-3 mean \pm SD approaches $2.77 \pm 0.57$ while in the inflatable expansion the TIMI stream range 1-3 mean \pm SD rises to $2.43 \pm 0.68$. That indicated huge factual contrast $(\mathrm{P}$ esteem $=0.044)$.In the emptied swell gathering the mean myocardial redden grade after the technique equivalent $2.63 \pm 0.67$ while in the inflatable expansion the mean myocardial become flushed evaluation equivalent $1.93 \pm 0.98$. That indicated profoundly critical factual distinction ( $\mathrm{P}$ esteem $=0.002$ ).

As respect the complexity in the collapsed swell gathering was $13.3 \%$ while in the inflatable expansion bunch was $43.3 \%$ and that was factually critical (p esteem 0.010).Both hematoma and draining happened in $10.0 \%$ of patients of the emptied expand gathering while at the same time draining happened in $16.7 \%$ and hematoma happened in $26.7 \%$ and that indicated non huge measurable distinction.

CIN happened in one patient in the emptied swell gathering $(3.3 \%)$ while it happened in 5 patients in the inflatable expansion gathering (16.7\%) that demonstrated non huge factual distinction. Cardiovascular breakdown side effects were introduced in just a single patient of the flattened inflatable gathering $(3.3 \%)$ versus 6 patients of the inflatable swelling gathering $(20.0 \%)$ that was factually critical (p value $=0.044$ ).

The pace of no reflow and distal embolization during PPCI was $6.7 \%$ in the collapsed expand gathering (in 2 patients) while it was $26.7 \%$ in the inflatable expansion gathering (in 8 patients). This indicated factually critical contrast ( $\mathrm{p}$ esteem 0.038). There were no factual differences between stents used during the study.

Table (1) Comparison between studied groups regarding the balloon size, TIMI flow and myocardial blush grade in the study groups.

\begin{tabular}{|c|c|c|c|c|c|c|}
\hline & & Deflated Balloon group & Balloon Inflation & Test value & P-value & Sig. \\
\hline & & No. $=30$ & No. $=30$ & & & \\
\hline \multirow[t]{2}{*}{ Balloon } & Deflated & $30(100.0 \%)$ & $0(0.0 \%)$ & $60.000 *$ & 0.000 & HS \\
\hline & Infaltion & $0(0.0 \%)$ & $30(100.0 \%)$ & & & \\
\hline \multirow[t]{7}{*}{ Size of balloon } & $1.25 \times 15$ & $2(6.7 \%)$ & $3(10.0 \%)$ & $20.630 *$ & 0.056 & NS \\
\hline & $1.25 \times 20$ & $1(3.3 \%)$ & $5(16.7 \%)$ & & & \\
\hline & $1.5 \times 15$ & $1(3.3 \%)$ & $3(10.0 \%)$ & & & \\
\hline & $1.5 \times 20$ & $1(3.3 \%)$ & $2(6.7 \%)$ & & & \\
\hline & $1.5 \times 5$ & $2(6.7 \%)$ & $0(0.0 \%)$ & & & \\
\hline & $2.15 \times 20$ & $1(3.3 \%)$ & $0(0.0 \%)$ & & & \\
\hline & $2.25 \times 15$ & $0(0.0 \%)$ & $1(3.3 \%)$ & & & \\
\hline
\end{tabular}


Table (1) Continue

\begin{tabular}{|c|c|c|c|c|c|c|}
\hline & $2.5 \times 10$ & $2(6.7 \%)$ & $1(3.3 \%)$ & & & \\
\hline & $2.5 \times 15$ & $5(16.7 \%)$ & $0(0.0 \%)$ & & & \\
\hline & $2.5 \times 20$ & $3(10.0 \%)$ & $1(3.3 \%)$ & & & \\
\hline & $2 \times 10$ & $4(13.3 \%)$ & $0(0.0 \%)$ & & & \\
\hline & $2 \times 20$ & $5(16.7 \%)$ & $8(26.7 \%)$ & & & \\
\hline & $2 \times 15$ & $3(10.0 \%)$ & $5(16.7 \%)$ & & & \\
\hline & $2 \times 12$ & $0(0.0 \%)$ & $1(3.3 \%)$ & & & \\
\hline TIMI flow & $\begin{array}{c}\text { Mean } \pm \text { SD } \\
\text { Range }\end{array}$ & $\begin{array}{c}2.77 \pm 0.57 \\
1-3\end{array}$ & $\begin{array}{c}2.43 \pm 0.68 \\
1-3\end{array}$ & $2.062 \bullet$ & 0.044 & $S$ \\
\hline MYO blush & $\begin{array}{l}\text { Meanness } \\
\text { Range }\end{array}$ & $\begin{array}{c}2.63 \pm 0.67 \\
1-3\end{array}$ & $\begin{array}{c}1.93 \pm 0.98 \\
0-3\end{array}$ & 3.231 • & 0.002 & HS \\
\hline
\end{tabular}

P-value >0.05: Non significant (NS); P-value <0.05: Significant (S); P-value< 0.01: highly significant (HS)

*:Chi-square test; $\bullet$ : Independent t-test

Table (2) Comparison between studied groups regarding MACE procedure difference, CIN, HF, complications among study groups, rate of aggrastate use and stent used during the study.

\begin{tabular}{|c|c|c|c|c|c|c|c|c|}
\hline & & \multicolumn{2}{|c|}{ Deflated Balloon } & \multicolumn{2}{|c|}{ Balloon Inflation } & \multirow[t]{2}{*}{ Test value* } & \multirow[t]{2}{*}{ P-value } & \multirow[t]{2}{*}{ Sig. } \\
\hline & & No. & $\%$ & No. & $\%$ & & & \\
\hline \multirow[t]{2}{*}{ MACE Procudure } & No & 29 & $96.7 \%$ & 22 & $73.3 \%$ & 6.405 & 0.011 & $S$ \\
\hline & Yes & 1 & $3.3 \%$ & 8 & $26.7 \%$ & & & \\
\hline \multirow[t]{2}{*}{ Re infarc,rehosp, death, } & No & 30 & $100.0 \%$ & 27 & $90.0 \%$ & 3.158 & 0.076 & NS \\
\hline & Yes & 0 & $0.0 \%$ & 3 & $10.0 \%$ & & & \\
\hline \multirow[t]{2}{*}{1 MONTH MACE } & No & 30 & $100.0 \%$ & 26 & $86.7 \%$ & 4.286 & 0.038 & $S$ \\
\hline & Yes & 0 & $0.0 \%$ & 4 & $13.3 \%$ & & & \\
\hline \multirow[t]{2}{*}{ Complication } & No & 26 & $86.7 \%$ & 17 & $56.7 \%$ & 6.648 & 0.010 & $S$ \\
\hline & Yes & 4 & $13.3 \%$ & 13 & $43.3 \%$ & & & \\
\hline \multirow[t]{2}{*}{ Bleeding } & No & 27 & $90.0 \%$ & 25 & $83.3 \%$ & 0.577 & 0.448 & NS \\
\hline & Yes & 3 & $10.0 \%$ & 5 & $16.7 \%$ & & & \\
\hline \multirow[t]{2}{*}{ Hematoma } & No & 27 & $90.0 \%$ & 22 & $73.3 \%$ & 2.783 & 0.095 & NS \\
\hline & Yes & 3 & $10.0 \%$ & 8 & $26.7 \%$ & & & \\
\hline \multirow{2}{*}{$\begin{array}{l}\text { GPIIb,IIIa inhibitor } \\
\text { (aggrastat use) }\end{array}$} & No & 26 & $86.7 \%$ & 22 & $73.3 \%$ & 1.667 & 0.197 & NS \\
\hline & Yes & 4 & $13.3 \%$ & 8 & $26.7 \%$ & & & \\
\hline \multirow[t]{2}{*}{ CIN } & No & 29 & $96.7 \%$ & 25 & $83.3 \%$ & 2.963 & 0.085 & NS \\
\hline & Yes & 1 & $3.3 \%$ & 5 & $16.7 \%$ & & & \\
\hline \multirow[t]{2}{*}{ HF } & No & 29 & $96.7 \%$ & 24 & $80.0 \%$ & 4.043 & 0.044 & $S$ \\
\hline & Yes & 1 & $3.3 \%$ & 6 & $20.0 \%$ & & & \\
\hline \multirow{2}{*}{$\begin{array}{l}\text { No reflow/ distal } \\
\text { emolization }\end{array}$} & No & 28 & $93.3 \%$ & 22 & $73.3 \%$ & 4.320 & 0.038 & S \\
\hline & Yes & 2 & $6.7 \%$ & 8 & $26.7 \%$ & & & \\
\hline \multirow[t]{6}{*}{ Stent } & Promus premier & 3 & $10.0 \%$ & 5 & $16.7 \%$ & 6.056 & 0.301 & NS \\
\hline & Orsiro & 10 & $33.3 \%$ & 8 & $26.7 \%$ & & & \\
\hline & Xience xpedition & 9 & $30.0 \%$ & 9 & $30.0 \%$ & & & \\
\hline & Resolute integrity & 8 & $26.7 \%$ & 4 & $13.3 \%$ & & & \\
\hline & Promus element & 0 & $0.0 \%$ & 3 & $10.0 \%$ & & & \\
\hline & Ultimaster & 0 & $0.0 \%$ & 1 & $3.3 \%$ & & & \\
\hline
\end{tabular}

P-value >0.05: Non significant (NS); P-value <0.05: Significant (S); P-value< 0.01: highly significant (HS) *:Chi-square test

\section{Discussion}

Major unfriendly heart occasion (MACE) was characterized as the composite pace of cardiovascular passing, MI and TLR. TIMI stream reviewing and myocardial redden grade (MBG) were utilized together to characterize angiographic no reflow as TIMI stream $<3$ (with any MBG evaluation) or TIMI stream 3 with MBG 0 - 1 [8].

In the current investigation, the utilization of the emptied swell direct stenting DBDS strategy can permit the representation of the downstream corridor and appraisal of the guilty party injury and henceforth more as often as possible permit DS.

The TIMI stream in the wake of utilizing of the flattened inflatable direct stenting DBDS strategy was higher mean $\pm \mathrm{SD}$ equivalent $2.77 \pm 0.57$ while in the inflatable inflation(pre-dilatation) bunch the TIMI stream equivalent $2.43 \pm 0.68$. That demonstrated huge factual distinction $(\mathrm{P}$ esteem $=0.044)$ and that was measurably huge.

The mean myocardial become flushed evaluation subsequent to utilizing of the collapsed expand direct 
stenting DBDS strategy was better equivalent $2.63 \pm$ 0.67 while in the inflatable expansion the mean myocardial redden grade equivalent $1.93 \pm 0.98$ and that was factually critical ( $\mathrm{P}$ esteem $=0.002$ ).

As respect MACE rate during the flattened inflatable method was fundamentally lower than MACE rate in inflatable expansion gathering (3.3\% Vs $26.7 \% \mathrm{P}$ value $=0.011$.

1 Month MACE in the inflatable expansion bunch was altogether higher $(13.3 \%)$ while in emptied swell gathering $(0.0 \%)$ ( $\mathrm{p}$ esteem 0.038$)$.

The pace of complexities in the flattened inflatable gathering was $13.3 \%$ while in the inflatable swelling bunch was $43.3 \%$ and that was factually critical ( $p$ esteem 0.010).

Cardiovascular breakdown happened in just a single patient of the flattened inflatable gathering (3.3\%) while it happened in 6 patients of the inflatable swelling gathering $(20.0 \%)$ that was measurably huge ( $\mathrm{p}$ value $=0.044)$.

The pace of no reflow and distal embolization after flattened inflatable direct stenting DBDS strategy was $6.7 \%$ while it was $26.7 \%$ in the inflatable swelling group.This demonstrated measurably critical distinction ( $\mathrm{p}$ esteem 0.038 )

Likewise, Bhupendra and associates [9] make a planned, observational, single-focus pilot concentrate from September 2016 to June 2018. More than 454 patients were tried out the examination. DS was performed when the offender vessel was pictured with in any event TIMI stream grade 1. Patients with complete impediment of the vessel after wire situation were exposed to emptied swell encouraged DS strategy (DBDS procedure) and DS was done at every possible opportunity.

DBDS strategy to encourage DS was fruitful in $68 \%$ patients (211/309). Last TIMI 3 stream was accomplished all the more habitually in the DS bunch when contrasted with PD gathering $(96.7 \%$ versus $92.3 \%, \mathrm{P}=0.04)$.

The procedural entanglements were likewise fundamentally lower in DS gathering $(0.6 \%$ versus $7.6 \%, \mathrm{P}<0.001)$.

At 30 days, the major antagonistic cardiovascular occasion (MACE) rate was fundamentally lower in the DS gathering $(2.4 \%$ versus $9.3 \%, \mathrm{P}=0.02)$, basically determined by lower rodent of target sore revascularization (TLR) $(0.9 \%$ versus $4.2 \%, \mathrm{P}=0.01)$.

The achievement pace of the strategy was in courses with $\leq 1$ TIMI grade stream after wire arrangement to accomplish. Generally speaking,. Difficulty perhaps identified with procedure was noted distinctly in one patient who built up no-reflow $(0.3 \%)$. This epic procedure seems, by all accounts, to be an attainable and safe alternative for encouraging DS of the guilty party vessel [10].

Different techniques used to improve ourtcomes of essential PCI in STEMI include:

\section{Blood clot desire during essential PCI}

The single-focus TAPAS preliminary enlisted 1071 patients with STEMI, demonstrating a pattern for a lessening in major antagonistic cardiovascular occasions (MACE) and furthermore showing a marginal endurance advantage in patients with STEMI randomized to the TA bunch contrasted and patients in the customary PCI bunch at 30 days (mortality: $2.1 \%$ [11 of 529] versus $4.0 \%$ [21 of 531]; hazard proportion $[\mathrm{RR}], \quad 0.52 ; 95 \%$ certainty span [CI], 0.26-1.07; $\mathrm{P}=0.07$; reinfarction: $0.8 \%$ [ 4 of 529] versus $1.9 \%$ [10 of 531]; RR, 0.40 ; 95\% CI, 0.13-1.27; P=0.11; targetvessel revascularization [TVR]: $4.5 \%$ [24 of 529] versus 5.8\% [31 of 531]; RR, 0.77; 95\% CI, 0.46-1.30; $\mathrm{P}=0.34$; MACE: $6.8 \%$ [36 of 529] versus $9.4 \%$ [50 of 531]; RR, 0.72; 95\% CI, 0.48-1.02; $\mathrm{P}=0.12$ ). The paces of death and MACE at 30 days were both altogether identified with myocardial redden grade (MBG), goal of ST-section height, and ST-fragment deviation $(\mathrm{P}=0.003$ for the relationship among death and $\mathrm{MBG})$, individually. One-year development of the TAPAS concentrate additionally uncovered that TA prior to stenting of the infarcted conduit fundamentally improved the clinical result contrasted and customary PCI [11].

\section{Distal assurance gadget}

Introductory Thrombolysis in Myocardial Infarction (TIMI) 0 or 1 stream was available in $67 \%$ of the embolic assurance gathering and $68 \%$ of the PCI alone gathering. Also, noticeable clots was available in $68 \%$ and $75 \%$, individually. There was no distinction in the essential result among any subgroup tried. There was no distinction in the greatest degree of creatine-kinase $(\mathrm{p}=0.99)$, troponin $\mathrm{T}(\mathrm{p}=0.87)$, or divider movement file $(\mathrm{p}=0.35)$ between the gatherings. There was additionally no distinction in 30-day major unfriendly cardiovascular occasions $(5.4 \%$ versus $3.2 \%, \mathrm{p}=0.17)$, separately for embolic security versus PCI alone. At 15 months, distinct stent apoplexy had happened in $2.9 \%$ of the embolic security bunch versus $0.3 \%$ of the PCI alone gathering $(\mathrm{p}=0.02)$ and target injury revascularization was $12.5 \%$ versus $7 \%(\mathrm{p}=0.02)$, this examination reason that among patients with STEMI, distal embolic insurance before PCI didn't diminish the rate of complete ST-fragment goal, most extreme degree of cardiovascular catalysts, divider movement file, or major unfriendly heart occasions contrasted and PCI alone. Truth be told, there were expanded unfriendly occasions late in development [12].

Intracoronary pharmacological specialists used to improve result of Primary PCI in STEMI: (adenosine, nitroprusside, diltiazem, verapamil and Fibrinolytic treatment).

Adenosine: Intracoronary adenosine treatment prompted altogether more post-PCI STRes [relative danger (RR) 1.39, 95\% certainty span (CI) 1.01-1.90; p $=0.04]$ and decrease in leftover ST fragment rise (RR 0.82, CI 0.69-0.99; p = 0.04) however didn't improve TIMI 3 stream (RR 1.09, CI 0.94-1.27; p = 0.25), 
MBG3 (RR 1.04, CI 0.65-1.69; p = 0.88), top CK-MB focus (mean distinction $-39.43, \mathrm{CI}-120.223$ to 41.371 ; $\mathrm{p}=0.339$ ) and post-PCI EF (mean contrast $1.238, \mathrm{CI}$ -5.802 to $8.277 ; \mathrm{p}=0.730)$. There was a pattern towards progress and MACE (RR 0.64, CI 0.40-1.03; $\mathrm{p}=0.06)$, rate of HF (RR 0.47, CI 0.19-1.12; p = 0.08) and CV mortality (RR 0.15 , CI $0.02-1.23$; p = 0.08) that didn't arrive at factual centrality yet no distinction altogether cause mortality (RR 0.77, CI 0.25-2.34; $\mathrm{p}=$ 0.64). Wellbeing investigation indicated no huge distinction in CP occasions (RR 1.26, CI 0.55-2.86; p $=0.58)$, bradycardia $(\operatorname{RR} 2.19$, CI $0.24-0.38 ; \mathrm{p}=0.49)$, VT (chances proportion 0.61, CI 0.08-4.90; $\mathrm{p}=0.64$ ) and VF (RR 0.49, CI 0.13-1.90; p $=0.30$ ), yet fundamentally more second-degree AVB (RR 7.88, CI 4.15-14.9; $\mathrm{p}<0.01$ ) in the adenosine bunch contrasted and the fake treatment gathering. Intracoronary adenosine organization was very much endured and essentially improved electrocardiographic results with a propensity towards progress in MACE, HF and CV mortality that couldn't arrive at measurable centrality [13].

Nitroprusside (NTP) : Intracoronary NTP altogether diminished the frequency of thrombolysis in myocardial dead tissue (TIMI) stream grade (TFG) $\leq 2$ (RR: 0.47, 95\% CI: 0.30-0.73, P =0.001); the adjusted TIMI outline tally (CTFC) (WMD: - 5.28, 95\% CI: 6.79 to $3.78, \mathrm{P}=0.000)$ expanded the occasions of myocardial become flushed evaluation $(M B G) \geq 2$ (RR: $1.12,95 \% \mathrm{CI}: 1.01-1.24, \mathrm{P}=0.038)$, and decreased the occurrence of major unfriendly heart occasions (MACE) (RR: 0.43, 95\% CI: 0.27-0.70, P = 0.001). Despite the fact that the occasions of the total ST fragment goal (STR) didn't arrive at factual centrality, there was a pattern showing improvement in the intracoronary NTP gathering (RR: 1.143, 95\% CI: $0.97-1.34, \quad P=0.101)$. Intracoronary NTP can altogether diminish the frequency of angiographic NR during PPCI, just as the occurrence of MACE. It is by all accounts a promising adjunctive treatment for NR during PPCI [14].

\section{Conclussion}

We presumed that DBDS strategy has a superior clinical outcomes,lower multi month MACE, lower complexities and lower distal embolization and no reflow rate. It has all the earmarks of being basic, achievable and safe. It helps in amplifying DS and could offer a choice to expand pre dilatation (PD) and goal thrombectomy in STEMI patients. Nonetheless, bigger examinations with longer development are required before a wider application of this technique.

\section{References}

[1] D.S. Menees, E.D.Peterson, Y.Wang, J.P.Curtis, Door-to-balloon time and mortality among patients undergoing primary PCI. N Engl J Med, Vol.369(10), PP.901-909,2013.

[2] E.C.Keeley, J.A.Boura, C.L.Grines, Primary angioplasty versus intravenous thrombolytic therapy for acute myocardial infarction: a quantitative review of 23 randomised trials. Lancet, Vol.361 (9351), PP.13-20,2003.

[3] A.Seto, M.Kern, Direct stenting for STEMI: does it really make a difference? Catheter Cardiovasc Interv, Vol.84(6), PP.932-933,2014.

[4] S.G.Ellis, M.Tendera, M.A.de Belder, A.J.van Boven, Facilitated PCI in patients with STelevation myocardial infarction. N Engl J Med, Vol.358(21), PP.2205-2217,2008.

[5] E .Barbato, J.Marco, W.Wijns, Direct stenting. Eur Heart J, Vol.24(5), PP.394-403,2003.

[6] A.Y.Elgendy, I.Y.Elgendy, A.N. Mahmoud, A.A.Bavry ,Long- term outcomes with aspiration thrombectomy for patients undergoing primary percutaneous coronary intervention: A metaanalysis of randomized trials. Clin Cardiol, Vol.40(8), PP.534-541, 2017.

[7] M.A.Magalhaes, S. Minha, T .Lhermusier, L.Pendyala, Does direct stenting with drug-eluting stents improve outcome? A meta-analysis of 10,900 patients. Catheter Cardiovasc Interv, Vol.90(2), PP.213-222, 2017.

[8] G.Niccoli, F.Burzotta, L.Galiuto, Myocardial noreflow in humans. J Am Coll Cardiol, Vol. 54(4), PP.281- 292,2009.

[9] Verma, Bhupendra , Singh, Amrita , Deflated Balloon-Facilitated Direct Stenting in Primary Angioplasty (The DBDS Technique): A Pilot Study. Cardiology Research, Vol. 9, PP. 284292,2018.

[10] G.Achkoutya, J.Guillaume, G.Dillinger, Microcatheter -Facilitated primary angioplasty in ST segment elevation myocardial infarction. 10.1016/j.cjca, Vol. 11, PP.005,2017.

[11]P.J.Vlaar, T. Svilaas, I.C.van der Horst, Cardiac death and reinfarction after 1 year in the thrombus aspiration during percutaneous coronary intervention in acute myocardial infarction study (TAPAS): a 1-year follow-up study. Lancet. vol.371, PP. 1915-1920.

[12]H.Kelbaek, C.J.Terkelsen, S.Helqvist, Randomized comparision of distal protection versus conventional treatment in primary percutaneous coronary intervention: The Drug Elution and Distal Protection in ST-Elevation Myocardial Infarction (DEDICATION) Trial. $J$ Am Coll Cardiol, Vol.51, PP.899-905,2008.

[13]M.Singh, T.Shah, Safety and efficacy of intracoronary adenosine administration in patients with acute myocardial infarction undergoing primary percutaneous coronary intervention: a meta-analysis of randomized controlled trials Ther Adv Cardiovasc Dis, Vol.6(3), PP. 101-114, 2012.

[14] S.Zhao, Q.Guoxian, T.Wen,Effect of intracoronary nitroprusside in preventing no reflow phenomenon during primary percutaneous coronary intervention: a meta-analysis. Interv Cardiol, Vol. 27(4), PP. 356-64,2014. 